\title{
Large Grain Superconducting RF Cavities at DESY
}

\author{
W. Singer, A. Brinkmann, A. Ermakov, J. Iversen, G. Kreps, \\ A. Matheisen, D. Proch, D. Reschke, X. Singer, M. Spiwek, H. Wen, \\ H. -G. Brokmeier \\ DESY, Notkestrasse 85, D-22607 Hamburg, Germany \\ GKSS, Max-Planck-Strasse, D-21502 Geesthacht, Germany
}

\begin{abstract}
The DESY R\&D program on cavities fabricated from large grain niobium explores the potential of this material for the production of approx. 1000 nine-cell cavities for the European XFEL. The program investigates basic material properties, comparing large grain material to standard sheet niobium, as well as fabrication and preparation aspects. Several singlecell cavities of TESLA shape have been fabricated from large grain niobium. A gradient up to 41 $\mathrm{MV} / \mathrm{m}$ at $\mathrm{Q} 0=1.4 \cdot 1010(\mathrm{~TB}=2 \mathrm{~K})$ was measured after electropolishing. The first three large grain nine-cell cavities worldwide have been produced under contract of DESY with ACCEL Instruments $\mathrm{Co}$. The first tests have shown that all three cavities reach an accelerating gradient up to $30 \mathrm{MV} / \mathrm{m}$ after BCP (Buffered Chemical Polishing) treatment, what exceeds the XFEL requirements for $\mathrm{RF}$ test in the vertical cryostat.
\end{abstract}

Keywords: Cavity, single crystal, accelerating gradient, material properties.

PACS: 29.17.+w, 85.25.-j, 65.40.-b

\section{INTRODUCTION}

The power of the niobium melting equipment is growing at the industry steadily. Ingots of high purity niobium with diameters of $300-500 \mathrm{~mm}$ can be produced today. In principle such large diameters allow one to simply slice discs of appropriate thickness from the ingot and the produce cavities by common deep drawing and electron beam welding techniques. This fabrication approach, producing "large-grain cavities", was proposed in joint work of JLab and CBMM and may be a cost effective option. Several single cell cavities produced at JLab from large grain high purity niobium demonstrated very good performance [1-4].

The main aim of the DESY R\&D program is to check, whether large grain cavities are an option for the fabrication of approximately 1000 nine-cell cavities for the European XFEL [5]. Two aspects have been pursued: the fabrication and preparation procedure, and the basic understanding of the difference between large grain (several or even one single crystal) and fine grain (polycrystalline) material and analysis of large grain perspectives as a SRF material.

\footnotetext{
CP927, Single Crystal-Large Grain Niobium Technology, International Niobium Workshop edited by G. R. Myneni, T. Carneiro, and A. Hutton

(C) 2007 American Institute of Physics 978-0-7354-0437-3/07/\$23.00
} 


\section{MATERIAL}

All the companies producing high purity niobium were invited to participate in the DESY large grain R\&D program. A big significance has the cost item. On the first glance the large grain option seems to be cost effective, because the long fabrication chain from ingot to fine grain sheet (forging, cleaning, rolling, cleaning, intermediate annealing, rolling, cleaning, final annealing) will be replaced by one step (cut the disc from ingot).

However cost effective cut the discs keeping tight thickness tolerances, purity and high surface quality is a challenge. Applying up to now EDM (electric discharge machining) or sawing with subsequent machining can not withstand the competition with the conventional sheet fabrication. Fa. HERAEUS put a lot of effort into the development of a cost effective cut procedure of the large grain discs and as it seems solved this problem. A machine available at semiconductor industry for cut of many discs simultaneously by wire sawing was adapted. The cutting conditions were developed so far that the discs slicing can be done now without polluting of the material [6]. The surface roughness of delivered discs met the specification and the thickness tolerances within $+/-50 \mu \mathrm{m}$, even better as DESY specification required.

Large grain niobium discs with $R R R=320-500$ for 10 single cell and three 9 cell cavities of TESLA shape were purchased by Fa. HERAEUS. The discs for cavities $1 \mathrm{AC} 3-1 \mathrm{AC} 5,1 \mathrm{DE} 20,1 \mathrm{DE} 21, \mathrm{AC} 112$ were annealed at the company at $1200^{\circ} \mathrm{C}$ for 6 hours with titanium and thereafter a layer of $0.5 \mathrm{~mm}$ was removed by machining from each site; the discs for remaining cavities were cut from ingot without additional treatment. Material for 3 single cell cavities was supplied by Fa. NINGXIA. In addition JLab and NPC (Niobium Products Company, Germany) provided us kindly with some large grain disks of $C B M M(R R R=250)$.

\begin{tabular}{|l|l|l|}
\hline $\begin{array}{l}\text { FIGURE 1a. Crystals distribution } \\
\text { on the CBMM disc and } \\
\text { measurement locations. }\end{array}$ & $\begin{array}{l}\text { FIGURE 1b. X-Ray } \\
\text { reflexes on central crystal } \\
\text { K1 of CBMM disc. (111) } \\
\text { orientation. }\end{array}$ & $\begin{array}{l}\text { FIGURE 1c. Example of reflex } \\
\text { splitting on the edge crystal K2 of } \\
\text { CBMM disc. }\end{array}$ \\
\hline
\end{tabular}




\begin{tabular}{|l|l|l|}
\hline $\begin{array}{l}\text { FIGURE } \\
\text { distribution on the HERAEUS } \\
\text { disc and measurement locations. }\end{array}$ & $\begin{array}{l}\text { FIGURE 2b. Pole figure of } \\
\text { the central crystal K1 of } \\
\text { HERAEUS disc. (100) } \\
\text { orientation (ideal cube } \\
\text { position rotated by 18 and } \\
\text { tilted by } 5^{\circ} \text { ). }\end{array}$ & $\begin{array}{l}\text { FIGURE 2c. Pole figure of the } \\
\text { crystals K2 -K5 of HERAEUS } \\
\text { orientation (100)). }\end{array}$ \\
\hline
\end{tabular}

The crystallographic structure of main large grains of three $\mathrm{Nb}$ discs of suppliers HERAEUS, CBMM und Ningxia was investigated. Measurements of single crystal reflexes by complete penetration and thereafter the orientation definition were done at DESY HASYLAB on instrument BW5 measurements volume was $1 \mathrm{~mm} 2 \times$ disc thicknesses). Normally the standard fine grain niobium sheets are produced on the way that they almost do not have preferred crystal orientation (texture); this caused the significant difference between large grain and fine grain material. Another difference consists of the distinction in grain boundaries. For example a fine grain $\mathrm{Nb}$ sheet corresponds the grain boundaries length $\sim 3000 \mathrm{~m}$, large grain $\mathrm{Nb}$ disc corresponds the length $\sim 3 \mathrm{~m} \mathrm{[6].}$

Are the big grains a real single crystals, what is the orientation in the main large grains and does the orientation influences the cavity performance were the main questions.

It turned out that the central crystal of CBMM ingot possesses very large homogeneity Fig. 1 a-c with the crystal orientation (111). Measurement in 7 different locations of the central crystal has shown very small fluctuations proving high quality of the single crystal. The edge crystals consist of many small single crystals and subcrystals. The reflexes are separated in several spots (Fig. 1c).

HERAUS disc consists of a big central crystal and some smaller edge crystals (Fig. $2 \mathrm{a}$-c). Central crystal has orientation of (100) close to ideal cube position (see pole Fig. 2b). Figure represents the superimposition of 5 measured locations $(1-4 ; 10)$ on the central crystal, what confirm the high quality of single crystal (weakly pronounced mosaic structure). The remaining crystals (single crystals of high quality too) show distinctly different orientations compare to the central crystal (Fig. 2c).

Ningxia disc consists of many crystals with wide scattering of the orientations, whereas are (111) orientation as well as (100). In a disc centre a signal splitting of reflexes (indication for several single crystals) and Debye Scherrer rings (indication for the powder or small crystals material) have been clearly observed in addition to the strong single crystal signal.

Bulk niobium cavities made from large grain or single crystal niobium may benefit from the thermal conductivity enhancement at around the running temperature $1.8 \mathrm{~K}$ 
due to the so-called 'phonon peak'. The total heat conductivity of the superconducting metal is obtained by adding the electron term and the phonon term.

$$
\lambda_{s}(T)=R(y)\left[\frac{\rho_{295 K}}{L_{0} \cdot R R R \cdot T}+a T^{2}\right]^{-1}+\left[\frac{1}{D \exp (y) T^{2}}+\frac{1}{B l T^{3}}\right]^{-1}
$$

$T$-Temperature, $R R R$-residual resistivity ratio, $l$-phonon mean free path. The fitting for high purity niobium coefficients are given in work [7].

It is to expect that contribution into thermal resistance from scattering of phonons on the grain boundaries are disappeared or will be significantly reduced due to reduced number of grain boundaries for large grain or missing of them for single crystal. Calculation of thermal conductivity using the formula for $\lambda s(T)$ and as the phonon mean free path the sample width of ca. $3 \mathrm{~mm}$ (instead of grain size for polycrystalline material normally ca. 50 $\mu \mathrm{m}$ ) can be seen in Fig. 3-4. Phonon peak is clearly pronounced.

The thermal conductivity of a series of fine grain (polycrystalline), large grain and single crystal niobium samples were measured at low temperatures. The experimental results (Fig. 3-4) are emphasis pronounced phonon peaks on large grain and single crystal heat treated at $800^{\circ} \mathrm{C}$ niobium samples produced by W.C. Heraeus (Figure 3), while no 'phonon peak' on fine grain samples independently on RRR value are observed.

No "phonon peak" was observed on a large grain niobium sample from Ningxia. This result is in correlation with presented above crystallographic structure investigation indicating that NINXIA large grains consist of many small powders like crystals.

Detected "phonon peak" is very susceptible to internal stress. Already $8.5 \%$ plastic deformation of the sample leads to complete destroying of the "phonon peak" (Fig.4). Similar results can be also found in reference [8]. It implies that the final annealing of cavity from large grain niobium at ca. $800^{\circ} \mathrm{C}$ is necessary not only from hydrogen outgassing point of view, but also it will support the thermal conductivity enhancement due to relax of the stress appeared during cavity fabrication.

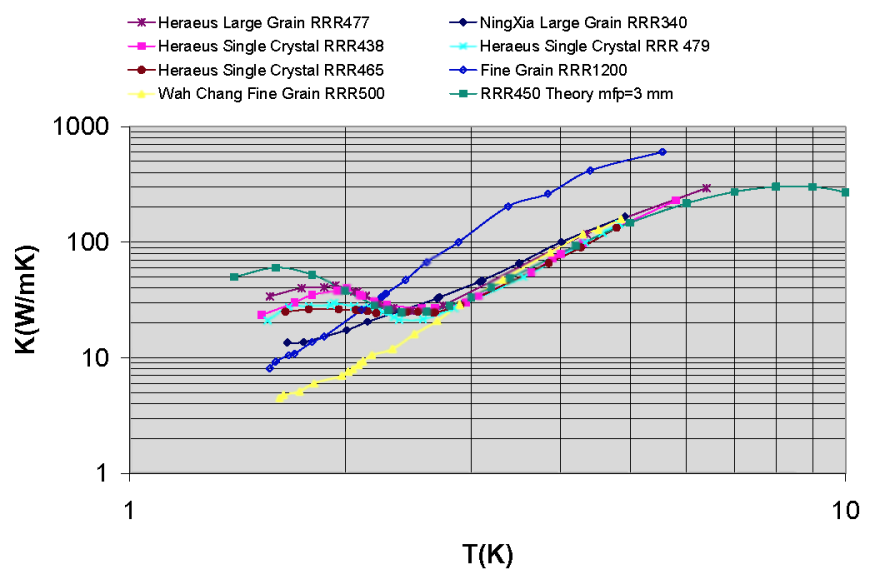

FIGURE 3. Thermal Conductivity of Fine Grain, Large Grain and Single Crystal Niobium (mfp: phonon mean free path). 


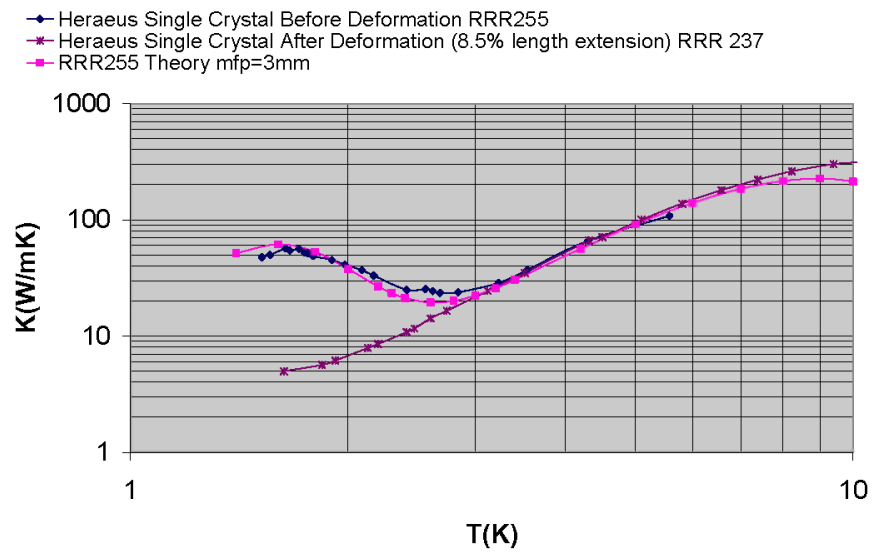

FIGURE 4. Plastic deformation influence on the thermal conductivity of single crystal niobium (mfp: phonon mean free path).

It is to be expected that the discs material from ingot is less vulnerable to foreign material inclusion and another types defects, because it is taken directly from many time re-melted homogeneous ingot material. Several sources of the material pollution like forging or rolling are not present in this case compare to fine grain sheets. The main discs used for cavity fabrication were scanned by eddy current device available at DESY. No any indications for localized defects in the crystals were observed (as example see Fig. 5) The grain boundaries have been distinctly seen what is sooner caused by sharp edges on grain boundaries after BCP etching as by impurities.

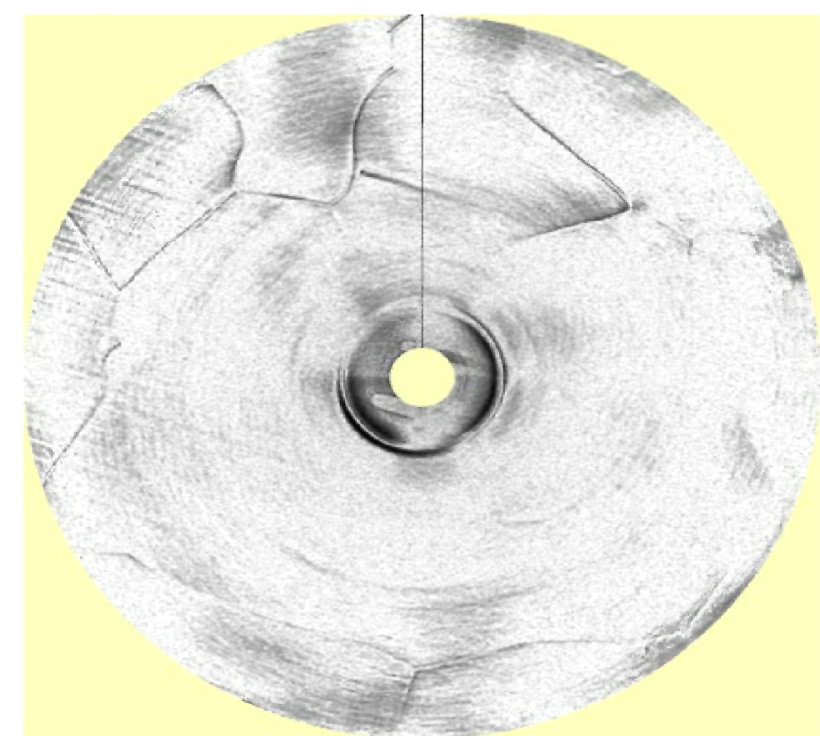

FIGURE 5. Eddy current image of the HERAEUS large grain disc. 


\section{FABRICATION}

Single cell cavity fabrication was done in two ways. Cavities 1AC3, 1AC4, 1AC5 and $1 \mathrm{AC7}$ were produced under a DESY contract with ACCEL Instruments Co., cavities 1DE20-1DE26 are planned to be welded on DESY electron beam equipment. Three nine cell cavities AC112 - AC114 (world wide first nine cell large grain cavities) were produced from HERAEUS material at Fa. ACCEL. Cavities 1AC6 and $1 \mathrm{AC} 8$ are produced from single crystal material. This item will be discussed separately.

Deep drawing of the half cell was done on the same way as for fine grain material using male and female tools (instead of female tool a rubberized material was used). All half cells were leak tight, but the grain boundaries were noticeably pronounced with steps up to $0.5 \mathrm{~mm}$. It turned out that the steps on grain boundaries can be reduced by applying spinning for half cell fabrication. Half cells for the cavity $1 \mathrm{AC} 5$ have been produced by this means. The steps on grain boundaries were less pronounced, but nevertheless clearly visible.

Optical coordinate measurement and creation of a 3D image was applied for estimation of the shape accuracy. Measurements were done at the Fa. DECOM with measurement accuracy of $10 \mu \mathrm{m}$ on half cells directly after deep drawing, on dumb bells with welded stiffening rings and on end half cell units with welded connecting flanges.

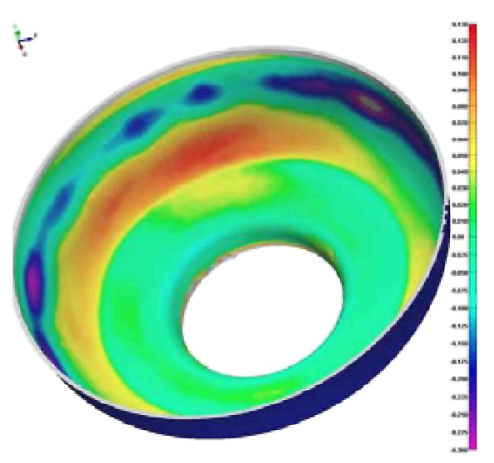

FIGURE 6. 3D Image of the fine grain long half cell No. her97; realized accuracy $+0,13 /-0,30 \mathrm{~mm}$.

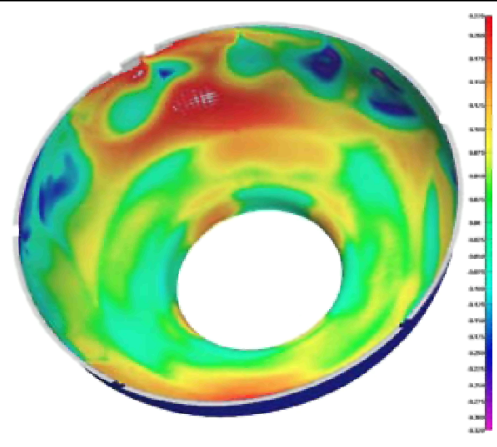

FIGURE 7. 3D Image of the large grain long half cell No. H069; realized accuracy $+0,22 /$ $0,32 \mathrm{~mm}$.

An example of the half cell shape in comparison with a standard fine grain niobium can be seen in Fig. 6-7. The large grains are fractionally pronounced (Fig.7). Variation of the large grain half cell shape is slightly bigger and partially does not meet the specified for Tesla Test Facility cavity tolerances ( $0.4 \mathrm{~mm}$ band width).

RF frequency measurement (Fig. 8) shows bigger deviation from expected frequency in large grain end cells compare to fine grain, what has according to a company statement sooner to do with tooling problems. Frequency deviation and standard deviation for large-grain middle half cells are smaller than for conventional material parts. With appropriate trimming the correct cavity length and the frequency 
of the fundamental mode in the nine cell cavities AC112-AC114 was achieved without any problems. The deep drawing behaviour for large grain is different compare to fine grain sheets (different spring back) but it is more stable and allows producing more uniform half cells. Better conformity in the shape to the specification requirements could be reached by the correction of the deep drawing tools. It has to be mentioned that a possession of a big central crystal is essential for large grain discs, otherwise forming of the iris can be critical (necking of the material in the iris area is difficult to avoid).

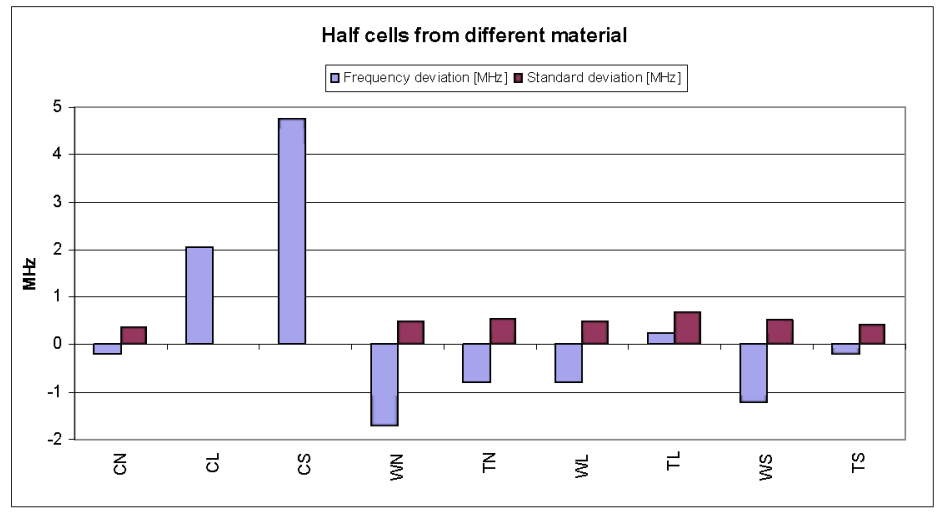

FIGURE 8. Frequency measurements of 6 end half cells ( $L$ and $S)$ and 48 middle half cells $(N)$ for cavities AC112-114. C - large grain, W - Wah Chang, T - Tokyo Denkai.

\section{PREPARATION AND RF TESTS}

The following aspects have to be considered in defining the preparation recipe for large grain cavities and deciding whether to use electropolish (EP) or the less challenging buffered chemical polish (BCP). On one hand after appropriate BCP the surface of the large grains themselves becomes very smooth and shiny, and the surface roughness is less than that achieved on fine-grain material after EP [2,9]. On the other hand the influence of the grain boundaries on the cavity performance is not sufficiently investigated. After EP, steps on grain boundaries are reduced, in contrast to grain boundary step size increase after BCP [9]. Both treatments are in progress in the DESY R\&D.

Applied treatment and some RF test results are summarized in table 1 . The best accelerating gradient of ca. $41 \mathrm{MV} / \mathrm{m}$ was achieved at the cavity $1 \mathrm{AC} 3$ after $150 \mu \mathrm{m}$ $\mathrm{EP}, 800^{\circ} \mathrm{C} 2 \mathrm{~h}, 40 \mu \mathrm{m}$ EP, baking $120^{\circ} \mathrm{C} 48 \mathrm{~h}$, HPR and was limited by quench at the equator (Fig. 9). Performance of cavity 1AC5 with half cells produced by spinning was limited by quench at lower accelerating gradient compare to deep drawn single cells (1AC3, 1AC4) and is probably related to fabrication issues. At the moment both treatments (EP and BCP) have been applied only on few large grain single cell cavities. It seems that the EP works more efficiently. More than $10 \mathrm{MV} / \mathrm{m}$ were gain during EP treatment on earlier BCP treated cavities 1AC3, 1 AC4 (table 1). 


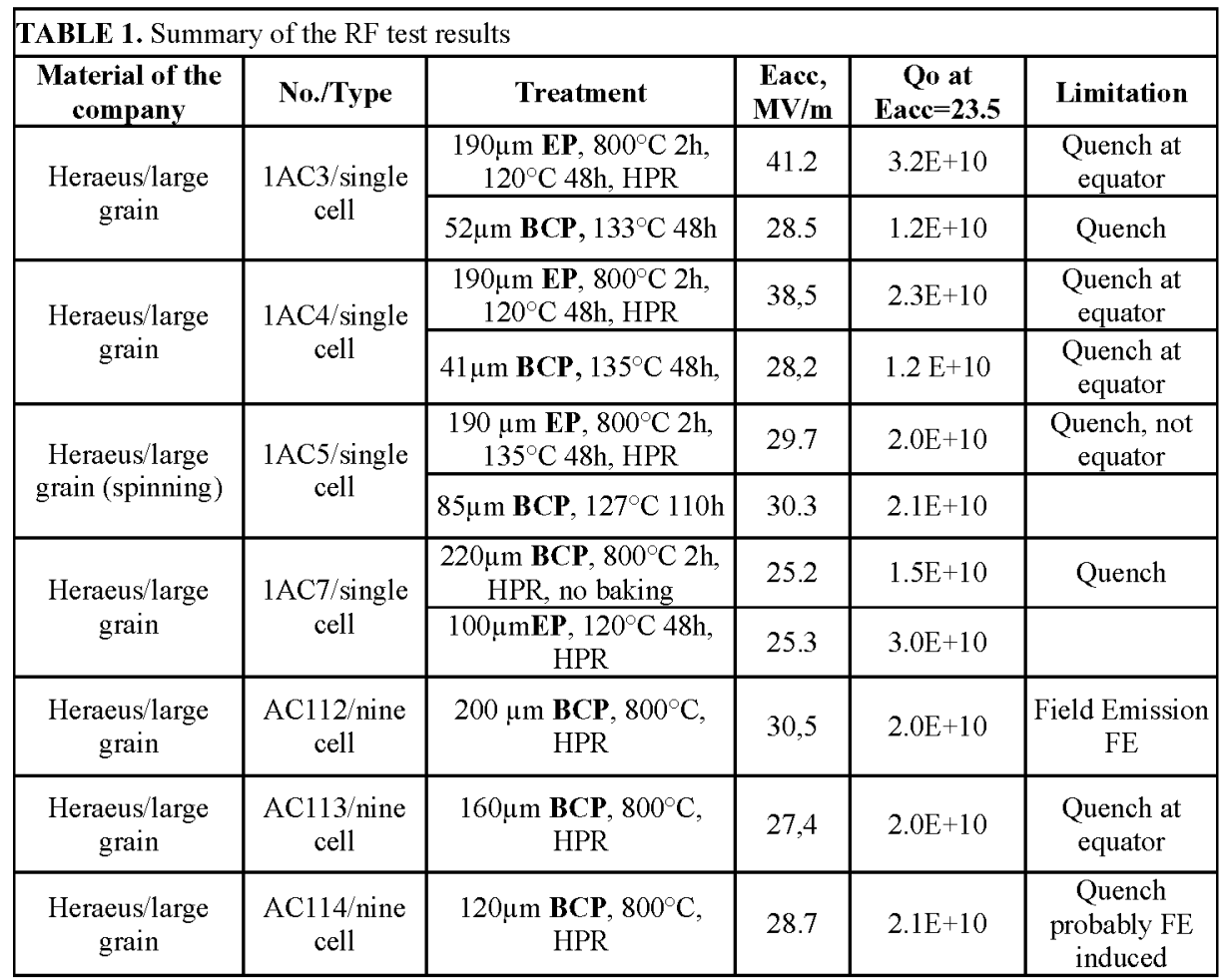

On three nine cell cavities $\mathrm{AC} 112-\mathrm{AC} 114$ up to now was done only first $\mathrm{RF}$ test after simple preparation of $120-200 \mu \mathrm{m} \mathrm{BCP}, 800^{\circ} \mathrm{C} 2 \mathrm{~h}, 20 \mu \mathrm{m}$ BCP, HPR (Table 1). The excitation curves can be seen in Fig. 10. The performance of $\mathrm{AC} 112$ and $\mathrm{AC} 114$ is restricted by some field emission. The performance of AC113 is limited by quench. $\mathrm{T}$ - Mapping inspections has detected the quench at the equator of cell 1 close to the area with a grain of comparatively small size ca. $2-3 \mathrm{~cm}$.

Achieved accelerating gradients up to $30 \mathrm{MV} / \mathrm{m}$ in three large grains TESLA shape cavities can be considered as a very good result. All three cavities meet the requirements of the XFEL specification $E a c c=25 \mathrm{MV} / \mathrm{m}$ with quality factor $\mathrm{Q}=1 \times 1010$ for the RF test in the vertical cryostat. It deserves to point out that the steps on grain boundaries were not grinded away.

An interesting comparison of these results with the performance of polycrystalline niobium cavities given similar treatment during the TTF project is presented in Fig. 11 .

It can be seen that the average value of Eacc for large grain cavities is almost by 5$7 \mathrm{MV} / \mathrm{m}$ higher compare to average Eacc of conventional cavities. One of plausible reasons of such differences could be the difference in the thermal conductivity caused by phonon peak. 


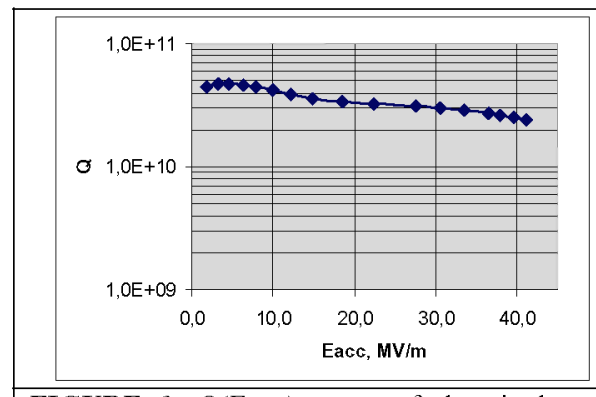

FIGURE 9. Q(Eacc) curve of the single cell cavity $1 \mathrm{AC} 3$ after EP treatment.

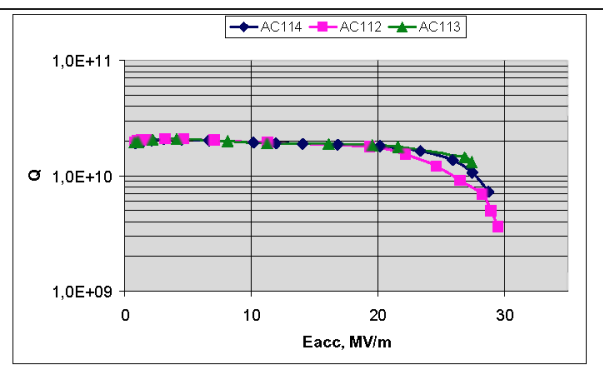

FIGURE 10. $Q$ (Eacc) curve of the nine cell cavity $\mathrm{AC} 112$ - AC114 after BCP treatment.

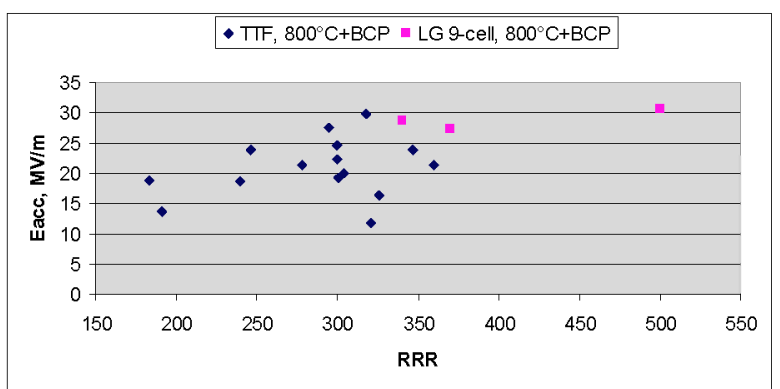

FIGURE 11. Comparison of large grain cavities RF results with performance of the cavities from polycrystalline niobium similarly treated in frame of TTF project.

\section{CONCLUSION}

Fabrication of cavities from large grain niobium deserves attention as potentially cost effective option with higher performance potential. Several companies are working now on development and optimization of the material fabrication. At least one vendor is currently in position to deliver such material in conformity with specification requirements and reasonable costs conditions.

Fabrication of single cell and multi cell cavities from large grain niobium has shown that no significant problem appeared during deep drawing and electron beam welding. The grain boundaries in the formed half cells are significantly pronounced (with steps up to a half of $\mathrm{mm}$ ).

Accelerating gradient similar to the best fine grain cavities can be achieved in large grain cavities especially after EP treatment. Very reasonable performance up to 30 $\mathrm{MV} / \mathrm{m}$ was achieved on the nine cell cavities after simple BCP preparation. The steps on grain boundaries were not ground away. Nevertheless surprisingly the cavity performance seems not to be essentially affected. A detailed analysis of the magnetic field enhancement in the case of large grain boundary steps would be very desirable. More statistics is necessary especially in order to compare the EP and BCP treatments.

It is expected that cavities made from large grain material, having reduced length of grain boundaries, will experience lower losses than fine grain material; thus higher Qo can be assumed. Shortage of data does not allow confirming this phenomenon. 
Investigations of the crystallographic structure allow concluding that despite in principle similar procedure of the ingot production the difference in the crystal orientation of ingots from different companies is significant. For example the main crystal of CBMM has the (111) orientation, while the main orientation of the central crystal of HERAEUS ingot is (100). The crystals quality is very different from company to company too.

No doubt that from orientation point of view as well as from grain boundary point of view more transparent effects can be observed on cavities produced from a single crystal. Both better understanding of the physics and better performance could be expected. The main problem on this way is how to produce single crystal of sufficient dimensions.

\section{ACKNOWLEDGMENTS}

We acknowledge all colleagues of DESY groups -MHF-SL-, -MKS-, -MPL-, MVA-, -MVP-, -ZM- for support in realization of the R\&D program. Many thanks to companies W.C. HERAEUS und ACCEL for the fruitful joint work on material and cavity fabrication, in particular to B. Spaniol, M. Pekeler and J. Schwellenbach. We are grateful to R. Brinkmann, P. Kneisel, D. Proch, and G. Rao Myneni for productive discussions.

\section{REFERENCES}

1. G. R. Myneni. Physical and Mechanical Properties of Single and Large Crystal High-RRR Niobium. Proceedings of $12^{\text {th }}$ International Workshop on RF Superconductivity (SRF 2005), July $10-15$, Ithaca, USA

2. P. Kneisel, G. R. Myneni, G. Ciovati, J. Sekutowicz, T.Carneiro. Performance of Large Grain and Single Crystal Niobium Cavities. Proceedings of $12^{\text {th }}$ International Workshop on RF Superconductivity (SRF 2005), July $10-15$, Ithaca, USA

3. P. Kneisel, G. R .Myneni, G. Giovati, J. Sekutowicz, T. Carneiro. Preliminary results from single crystal and very large crystal niobium cavities. Proceeding of of 2005 Particle Accelerator Conference, Knoxville, Tennessee, USA, Mai 16-20, 2005

4. P. Kneisel. Latest development in superconducting RF structures for beta $=1$ particle acceleration. Proceedings of 10th biennial European Particle Accelerator Conference, EPAC'06, Edinburgh, Scotland, 26 - 30 June, 2006

5. XFEL The European X-Ray Free-Electron Laser. Technical Design Report DESY 2006.

6. B. Spaniol. Proceedings of this Workshop, 2007.

7. F. Koechlin, B. Bonin, Parametrisation of the Niobium Thermal Conductivity in the Superconducting State, Supercond. Sci. Technol. 9 (1996)453-460.

8. W. Wasserbäch, Low-temperature thermal conductivity of plastically deformed niobium single crystal, Philosophical Magazine A, 1978, Vol.38, No.4, 401-431.

9. X. Singer, A. Brinkmann, H. G. Brokmeier, J. Iversen, P. Kneisel, G. R. Myneni, E. Schulz, W. Singer. INVESTIGATION OF INGOT MATERIAL WITH LARGE GRAIN FOR RF CAVITIES. Proceedings of $12^{\text {th }}$ International Workshop on RF Superconductivity (SRF 2005), July $10-15$, Ithaca, USA 